\title{
An improved approach for determining the optimal orientation of orthotropic material
}

\author{
H.C. Cheng, N. Kikuchi and Z.D. Ma \\ Department of Mechanical Engineering and Applied Mechanics, The University of Michigan, Ann Arbor, MI 48105, USA
}

\begin{abstract}
Several approaches for determining the optimal orientation in the topological optimization process are discussed in this paper. It is shown that the use of a strain based method may give an even worse result than the case in which the discrete orientational variables are fixed in the optimization process, because strong couplings exist among the orientational variables when the strain field is used. The stress field is less sensitive than the strain field with respect to the variations of the orientational variables. Therefore, the coupling between the orientational variables is relatively weak when the stress field is used. This explains why the stress based approaches (e.g. Suzuki and Kikuchi 1991; Díaz and Bendsøe 1992) are more efficient than the strain based method. This kind of approach will be generalized to deal with more general optimization problems. It will be shown that the new approach can not only obtain the best result for the problem as compared to the other methods, but also has the generality for various optimization problems. Several examples will be presented to support the above statements.
\end{abstract}

\section{Introduction}

The optimal topology design of structures has become a subject that calls for more and more attention of researchers and engineers in the field of structural optimization recently. Bends $\varnothing \mathrm{e}$ and Kikuchi (1988) introduced a Homogenization Based Optimization (HBO) method for finding optimal structures without the requirement of conjecturing the initial topologies. This method transforms the topological optimization problem into an Optimal Material Distribution (OMD) problem, where the composite material with many microscopical voids is introduced into the design problem (Fig. 1) so as to relax the original problem which was restricted to the use of an isotropic solid material. As was pointed out by Kohn (1986), the relaxed formulation has the advantage of obtaining fewer local minima, thus the global minima can be reached relatively easily. Such an idea is employed by Bends $\varnothing$ e and Kikuchi (1988), who employed a microstructure model for relaxing the problem as shown in Fig. 2, where three design variables, the sizes $a, b$ of the rectangular cavity and the orientation $\theta$ of the microstructure, are employed for a plane stress problem. Then the stiffness coefficients as well as the mass density of the structure are evaluated as the functions of these three design variables using the homogenization method. The optimization process is then used to obtain the OMD, which gives the optimal structure with the optimal topology.

There may exist a question: how important is the use of an orientational design variable for relaxing the problem?
Also, one might ask how a microstructure that forms an orthotropic material can be optimally oriented by a mathematical or physical consideration. This kind of problem can be generalized as an optimal orientation problem of orthotropic material, and was first discussed by Pedersen (1988). However, the discussion by Pedersen (1988) is limited to a unit cell case, in which the orientational variable is separated from the whole design domain to obtain the extreme strain energy density. This consideration is not sufficient for the real design problem, in which the orientational variable is a distributed parameter in the design domain, and the coupling exists among the orientational variables in two different spatial points of the design domain. Alternatively, Suzuki and Kikuchi (1991) indicated that the optimal orientation of the microstructure can be determined by the principal stress directions of the structure. This idea has been implemented in the HBO algorithm for solving the layout optimization problem. Later, Diaz and Bendsøe (1992) presented another approach dealing with the layout optimization problem under multi-loading. This approach is similar to that of Pedersen (1988), but instead of using the strain energy density, it employs the stress based formulation to calculate the optimal orientation. Apparently, the stress based approach can provide much better results than the strain based approach (see also Olhoff et al. 1992). Hence, it is very interesting to study the distinctions between these different approaches, essentially between the stress based and strain based approaches, and their rationalism, in determining the optimal orientation.

The goals of this paper are not only to clarify the questionable points stated above, but also to derive a general approach for determining the optimal orientation in dealing with various optimization problems. It will be shown that the stress field is less sensitive than the strain field with respect to the variation of the orientational variable. Therefore, the coupling between the discrete orientational variables is relatively weak when the stress field is used. This explains why the stress based approach, as employed by Suzuki and Kikuchi (1991) and Diaz and Bendsøe (1992), is more efficient than the strain based method. This kind of approach will be generalized in this paper to deal with more general optimization problems. A typical eigenvalue optimization problem is considered, but the approach can be applied to other optimization problems. It will be shown that the new approach is also more accurate and efficient in determining the optimal orientation than the previous methods. To substantiate the issues discussed herein, the results obtained by the new approach will be compared with those of the previous methods, 
i.e. the strain based method, the principal stress direction method, and the mathematical programming method.

\section{The homogenized properties of the composite ma- terial}

Bendsøe and Kikuchi (1988) introduced composite material to structural optimization problems by using a variable microstructure as shown in Fig. 1 , in which $\Omega\left(\Omega \subset R^{3}\right)$ stands for the structural domain, $\Gamma_{t}$ the traction boundary and $\Gamma_{d}$ the displacement boundary. For a plane stress problem, the microstructure is considered as a unit cell with a rectangular cavity inside the cell as shown in Fig. 2, where the parameters $a, b$ characterize the dimensions of the cavity and $\theta$ stands for the orientation of the microstructure. The solid part of the microstructure is formed by isotropic material with the elastic coefficient $E_{i j k \ell}^{0}$ and mass density $\rho_{0}$, which are constant in the solid part of the microscopic domain $Y$.

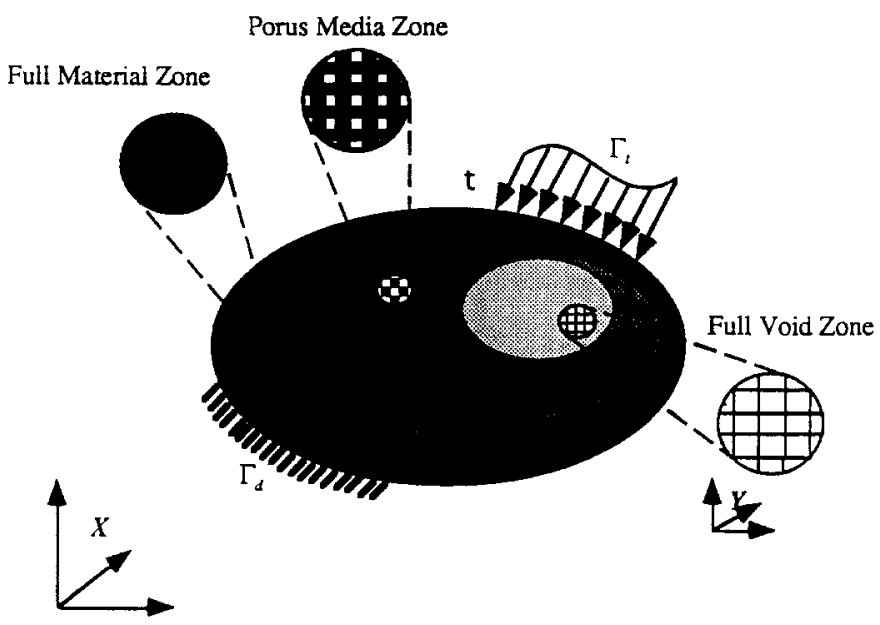

Fig. 1. Microstructures in a design domain

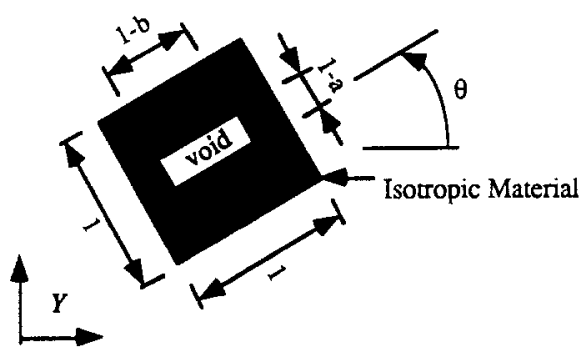

Fig. 2. A microstructure with design variables $a, b, \theta$

The linearly elastic problem for a dynamic system can be generally formulated using the weak form as

$$
\begin{aligned}
& \int_{\Omega} E_{i j k \ell}^{\varepsilon} \frac{\partial u_{i}^{\varepsilon}}{\partial x_{j}} \frac{\partial v_{k}}{\partial x_{\ell}} \mathrm{d} \Omega+\int_{\Omega} \rho^{\varepsilon} \frac{\partial^{2} u_{i}^{\varepsilon}}{\partial t^{2}} v_{i} \mathrm{~d} \Omega= \\
& \int_{\Omega} f_{i}^{\varepsilon} v_{i} \mathrm{~d} \Omega+\int_{\Gamma_{t}} t_{i} v_{i} \mathrm{~d} \Gamma, \quad \text { for } \forall \mathrm{v} \in V,
\end{aligned}
$$

where $u_{i}^{\varepsilon}$ stands for the displacement that yields equilibrium, $v_{i}$ the virtual displacement $\left(\mathbf{v}=\left\{v_{1}, v_{2}, v_{3}\right\}^{T}\right), f_{i}^{\epsilon}$ the body force, $t_{i}$ the boundary traction, and $V=\left\{\mathbf{v} \mid \mathbf{v} \in H^{1}(\Omega), \mathbf{v}=\right.$
0 on $\left.\Gamma_{d}\right\}$ stands for the space of kinematically admissible displacement fields, where $H^{1}(\Omega)$ is the Sobolev space. For the problem with the microstructure defined in Fig. 2, if we assume the orientation $\theta=0$ (i.e. the microstructure is not rotated), we have

$E_{i j k \ell}^{\varepsilon}=\left\{\begin{array}{ll}E_{i j k \ell}^{0} & \text { in solid } \\ 0 & \text { in cavity }\end{array}, \rho^{\varepsilon}= \begin{cases}\rho_{0} & \text { in solid } \\ 0 & \text { in cavity }\end{cases}\right.$

Using the homogenization method (e.g. Lions 1981; Guedes 1990; Bendsøe and Kikuchi 1988), (1) can be homogenized as

$$
\begin{aligned}
& \int_{\Omega} E_{i j k \ell}^{h} \frac{\partial u_{i}^{0}}{\partial x_{j}} \frac{\partial v_{k}}{\partial x_{\ell}} \mathrm{d} \Omega+\int_{\Omega} \rho^{h} \frac{\partial^{2} u_{i}^{0}}{\partial t^{2}} v_{i} \mathrm{~d} \Omega= \\
& \int_{\Omega} f_{i}^{h} v_{i} \mathrm{~d} \Omega+\int_{\Gamma_{t}} t_{i} v_{i} \mathrm{~d} \Gamma, \quad \text { for } \forall \mathbf{v} \in V,
\end{aligned}
$$

where, $u_{i}^{0}$ stands for the average displacements in the microstructural domain $Y, E_{i j k \ell}^{h}, \rho^{h}$ and $f_{i}^{h}$ are the homogenized elastic coefficients, mass density, and body force, respectively,

$$
\begin{aligned}
& E_{i j k \ell}^{h}=\frac{1}{|Y|} \int_{Y}\left(E_{i j k \ell}^{\varepsilon}-E_{i j p q}^{\varepsilon} \frac{\partial \chi_{p}^{k \ell}}{\partial y_{q}}\right) \mathrm{d} \mathbf{y}, \\
& \rho^{h}=\frac{1}{|Y|} \int_{Y} \rho^{\varepsilon} \mathrm{d} \mathbf{y} \\
& f^{h}=\frac{1}{|Y|} \int_{Y} f^{\varepsilon} \mathrm{d} \mathbf{y}
\end{aligned}
$$

where $|Y|$ stands for the volume (area) of $Y$, and $\chi_{p}^{k \ell}$ is the solution of the microscopic problem that characterizes the micromechanical behaviour of a specific microstructure, i.e.

$\int_{Y}\left(E_{i j k \ell}^{\epsilon}-E_{i j p q}^{\varepsilon} \frac{\partial \chi_{p}^{k \ell}}{\partial y_{q}}\right) \frac{\partial v_{i}}{\partial y_{j}} \mathrm{~d} \mathbf{y}=0, \quad$ for $\forall \mathbf{v} \in V_{Y}$,

where the space $V_{Y}=\left\{\mathbf{v} \mid \mathbf{v} \in H^{1}(Y)\right.$ and $\mathbf{v}$ is $Y$-periodic $\}$ is defined on the microstructural domain $Y$.

When the rotation of the microstructure is also considered as shown in Fig. 2, the rotated homogenized coefficients $E_{i j k \ell}^{H}$ and $\rho^{H}$ for the plane stress problem can be obtained as

$E_{i j k \ell}^{H}=\sum_{q=1}^{2} \sum_{p=1}^{2} \sum_{s=1}^{2} \sum_{r=1}^{2} c_{i q} c_{j p} c_{k s} c_{\ell r} E_{q p s r}^{h}$

and $\rho^{H}=\rho^{h}$,

where

$c_{11}=c_{22}=\cos \theta$ and $c_{21}=-c_{12}=\sin \theta$.

Finally, the weak form for the rotated system can be obtained as

$\int_{\Omega} E_{i j k \ell}^{H} \frac{\partial u_{i}^{0}}{\partial x_{j}} \frac{\partial v_{k}}{\partial x_{\ell}} \mathrm{d} \Omega+\int_{\Omega} \rho \frac{\partial^{2} u_{i}^{0}}{\partial t^{2}} v_{i} \mathrm{~d} \Omega=$

$\int_{\Omega} f_{i}^{h} v_{i} \mathrm{~d} \Omega+\int_{\Gamma_{t}} t_{i} v_{i} \mathrm{~d} \Gamma, \quad$ for $\forall \mathbf{v} \in V$. 
Here, it is obvious that the homogenized elastic coefficient $E_{i j k \ell}^{H}$ is a function of the design variables $a, b$ and $\theta$, and the homogenized mass density $\rho^{H}$ is a function of the design variables $a$ and $b$, i.e.

$E_{i j k \ell}^{H}=E_{i j k \ell}^{H}(a, b, \theta)$ and $\rho^{H}=\rho^{H}(a, b)$.

Note that the homogenized mass density is not a function of the orientational variable in this problem.

Using a matrix expression for the rotated elastic coefficient, we can define

$\mathbf{D}^{H}=\left[\begin{array}{lll}E_{1111}^{H} & E_{1122}^{H} & E_{1112}^{H} \\ E_{1122}^{H} & E_{2222}^{H} & E_{2212}^{H} \\ E_{1112}^{H} & E_{2212}^{H} & E_{1212}^{h}\end{array}\right]$.

Then the rotated elastic matrix is obtained as

$\mathbf{D}^{H}=\mathbf{D}^{0}+\mathbf{D}^{1} \sin 2 \theta+\mathbf{D}^{2} \cos 2 \theta+$

$\mathbf{D}^{3} \sin 2 \theta \cos 2 \theta-\mathbf{D}^{4} \sin ^{2} 2 \theta$,

where matrices $\mathbf{D}^{i}(i=0,1, \ldots, 4)$ are independent of the orientational variable $\theta$, and

$\mathbf{D}^{0}=\left[\begin{array}{ccc}\left(E_{1111}^{h}+E_{2222}^{h}\right) / 2 & E_{1122}^{h} & 0 \\ E_{1122}^{h} & \left(E_{1111}^{h}+E_{2222}^{h}\right) / 2 & 0 \\ 0 & 0 & E_{1212}^{h}\end{array}\right]$,

$\mathbf{D}^{1}=d_{1}\left[\begin{array}{lll}0 & 0 & 1 \\ 0 & 0 & 1 \\ 1 & 1 & 0\end{array}\right]$

$\mathbf{D}^{2}=2 d_{1}\left[\begin{array}{ccc}1 & 0 & 0 \\ 0 & -1 & 0 \\ 0 & 0 & 0\end{array}\right]$

$\mathbf{D}^{3}=d_{2}\left[\begin{array}{ccc}0 & 0 & 1 \\ 0 & 0 & -1 \\ 1 & -1 & 0\end{array}\right]$

$\mathbf{D}^{4}=d_{2}\left[\begin{array}{ccc}1 & -1 & 0 \\ -1 & 1 & 0 \\ 0 & 0 & -1\end{array}\right]$

and

$d_{1}=\frac{1}{4}\left(E_{1111}^{h}-E_{2222}^{h}\right)$,

$d_{2}=\frac{1}{4}\left(E_{1111}^{h}+E_{2222}^{h}-2 E_{1122}^{h}-4 E_{1212}^{h}\right)$.

\section{Sensitivity analysis}

First, we consider a static problem which minimizes the mean compliance of the structure. Thus the objective function is defined as

$\Pi=\int_{\Omega} E_{i j k \ell}^{H} \frac{\partial u_{i}^{0}}{\partial x_{j}} \frac{\partial u_{k}^{0}}{\partial x_{\ell}} \mathrm{d} \Omega$.

Operating a partial derivative with respect to the orientational variable $\theta$ on the objective function $\Pi$ gives

$\frac{\partial \Pi}{\partial \theta}=\int_{\Omega}\left[\frac{\partial E_{i j k \ell}^{H}}{\partial \theta} \frac{\partial u_{i}^{0}}{\partial x_{j}} \frac{\partial u_{k}^{0}}{\partial x_{\ell}}+2 E_{i j k \ell}^{H} \frac{\partial}{\partial \theta}\left(\frac{\partial u_{i}^{0}}{\partial x_{j}}\right) \frac{\partial u_{k}^{0}}{\partial x_{\ell}}\right] \mathrm{d} \Omega$.
Operating a partial derivative on the weak form (10) with respect to the orientational variable $\theta$ for the case $\rho^{H}=0$, we have

$\int_{\Omega}\left[\frac{\partial E_{i j k \ell}^{H}}{\partial \theta} \frac{\partial u_{i}^{0}}{\partial x_{j}}+E_{i j k \ell}^{H} \frac{\partial}{\partial \theta}\left(\frac{\partial u_{i}^{0}}{\partial x_{j}}\right)\right] \frac{\partial v_{k}}{\partial x_{\ell}} \mathrm{d} \Omega=0$.

Therefore,

$\int_{\Omega} E_{i j k \ell}^{H} \frac{\partial}{\partial \theta}\left(\frac{\partial u_{i}^{0}}{\partial x_{j}}\right) \frac{\partial u_{k}^{0}}{\partial x_{\ell}} \mathrm{d} \Omega=-\int_{\Omega} \frac{\partial E_{i j k \ell}^{H}}{\partial \theta} \frac{\partial u_{i}^{0}}{\partial x_{j}} \frac{\partial u_{k}^{0}}{\partial x_{\ell}} \mathrm{d} \Omega$.

Substituting (19) into (17), we can finally obtain the sensitivity of the mean-compliance with respect to the orientational variable $\theta$ as follows:

$\frac{\partial \Pi}{\partial \theta}=-\int_{\Omega} \frac{\partial E_{i j k \ell}^{H}}{\partial \theta} \frac{\partial u_{i}^{0}}{\partial x_{j}} \frac{\partial u_{k}^{0}}{\partial x_{\ell}} \mathrm{d} \Omega$.

It should be noted that if we assume the displacement field, i.e. $u_{k}^{0}$ is constant with respect to the orientational variable $\theta$, then from (17) we have

$\frac{\partial \Pi}{\partial \theta}=\int_{\Omega} \frac{\partial E_{i j k \ell}^{H}}{\partial \theta} \frac{\partial u_{i}^{0}}{\partial x_{j}} \frac{\partial u_{k}^{0}}{\partial x_{\ell}} \mathrm{d} \Omega \quad$ (for fixed $u_{k}^{0}$ ).

This result has an opposite sign to the result obtained in (20), and it was used by some researchers. However, (21) is incorrect when the displacement field is not fixed as in the usual structural design case.

For an eigenvalue problem, the weak form can be written as

$\int_{\Omega} E_{r s p q}^{H} \frac{\partial \phi_{r}^{i}}{\partial x_{s}} \frac{\partial v_{p}}{\partial x_{q}} \mathrm{~d} \Omega-\lambda_{i} \int_{\Omega} \rho^{H} \phi_{r}^{i} v_{r} \mathrm{~d} \Omega=0$,

for $\forall \mathbf{v} \in V$,

where $\lambda_{i}$ stands for the $i$-th eigenvalue, and $\phi^{i}$ the corresponding eigenvector. Operating a partial derivative on (22) gives

$\int_{\Omega}\left[\frac{\partial E_{r s p q}^{H}}{\partial \theta} \frac{\partial \phi_{r}^{i}}{\partial x_{s}} \frac{\partial v_{p}}{\partial x_{q}}+E_{r s p q}^{H} \frac{\partial}{\partial \theta}\left(\frac{\partial \phi_{r}^{i}}{\partial x_{s}}\right) \frac{\partial v_{p}}{\partial x_{q}}-\right.$

$\left.\frac{\partial \lambda_{i}}{\partial \theta} \rho^{H} \phi_{r}^{i} v_{r}-\lambda_{i} \rho^{H} \frac{\partial \phi_{r}^{i}}{\partial \theta} v_{r}\right] \mathrm{d} \Omega=0, \quad$ for $\forall \mathbf{v} \in V$.

If $v_{r}=\phi_{r}^{i}$ and we assume

$\int_{\Omega} \rho^{H} \phi_{r}^{i} \phi_{r}^{i} \mathrm{~d} \Omega=1$,

from (23), we have

$\frac{\partial \lambda_{i}}{\partial \theta}=\int_{\Omega}\left[\frac{\partial E_{r s p q}^{H}}{\partial \theta} \frac{\partial \phi_{r}^{i}}{\partial x_{s}} \frac{\partial \phi_{p}^{i}}{\partial x_{q}}+\right.$

$\left.E_{r s p q}^{H} \frac{\partial}{\partial \theta}\left(\frac{\partial \phi_{r}^{i}}{\partial x_{s}}\right) \frac{\partial \phi_{p}^{i}}{\partial x_{q}}-\lambda_{i} \rho^{H} \frac{\partial \phi_{r}^{i}}{\partial \theta} \phi_{r}^{i}\right] \mathrm{d} \Omega$.

Substituting $v_{r}=\partial \phi_{r}^{i} / \partial \theta$ into (22), we have 
$\int_{\Omega}\left[E_{r s p q}^{H} \frac{\partial}{\partial \theta}\left(\frac{\partial \phi_{r}^{i}}{\partial x_{s}}\right) \frac{\partial \phi_{p}^{i}}{\partial x_{q}}-\lambda_{i} p^{H} \frac{\partial \phi_{r}^{i}}{\partial \theta} \phi_{r}^{i}\right] \mathrm{d} \Omega=0$.

Therefore, finally the sensitivity of an arbitrary eigenvalue with respect to the orientational variable is

$\frac{\partial \lambda_{i}}{\partial \theta}=\int_{\Omega} \frac{\partial E_{r s p q}^{H}}{\partial \theta} \frac{\partial \phi_{r}^{i}}{\partial x_{s}} \frac{\partial \phi_{p}^{i}}{\partial x_{q}} \mathrm{~d} \Omega$.

\section{Previous methods for determining the optimal orientation}

The current problem is how to determine the orientational design variable $\theta$ for maximizing (or minimizing) the objective function when the sizing design variables $a$ and $b$ have been determined in the optimization process. The optimality criteria for the orientational variable can be written as

$\frac{\partial \Pi}{\partial \theta}=0$.

Several methods to solve the problem have been discussed, i.e. Pedersen's method (Pedersen 1988), the principal stress direction method (Suzuki and Kikuchi 1991), and a stress based method for the multi-loading case by Díaz and Bendsøe (1992).

\subsection{Pedersen's method}

Pedersen (1988) discussed this kind of problem using a fixed material and a rotating strain field. Pedersen (1988) considered the strain energy density, which is one half of the mean-compliance in a unit area. As a result, the sensitivity of the strain energy density with respect to the orientational variable obtained by Pedersen (1988) is

$\frac{\partial u}{\partial \theta}=$

$-2\left(\varepsilon_{I}-\varepsilon_{I I}\right) \sin 2 \theta\left[d_{1}\left(\varepsilon_{I}+\varepsilon_{I I}\right)+d_{2}\left(\varepsilon_{I}-\varepsilon_{I I}\right) \cos 2 \theta\right],(29)$

where $u$ stands for the strain energy density $\left(\Pi=2 \int_{\Omega} u \mathrm{~d} \Omega\right)$, and, $\varepsilon_{I}$ and $\varepsilon_{I I}\left(\varepsilon_{I} \geq \varepsilon_{I I}\right)$ are the principal strains. Solving $\partial u / \partial \theta=0$ by the use of (29), we have

$\sin 2 \theta=0$ or $\cos 2 \theta=-\frac{d_{2}\left(\varepsilon_{I}-\varepsilon_{I I}\right)}{d_{1}\left(\varepsilon_{I}+\varepsilon_{I I}\right)}$.

Several solutions can be obtained from solving (30). Here one needs to determine an optimal one for the problem. A detailed discussion about this determination can be found in the paper by Pedersen (1988).

Pedersen's results are limited in that the optimal orientation $\theta$ is determined for the extreme strain energy density at a special point of the design domain only. Also, it is assumed that the principal strains, $\varepsilon_{I}$ and $\varepsilon_{I I}$, as well as the principal strain directions, are constant with respect to the orientational variable. Indeed, as we have mentioned before, the principal strains are an implicit function of the orientational variable $\theta$. The principal strains, as well as the principal strain directions, can be changed when the orientational variable $\theta$ is varied. These changes (in the principal strains and their directions) can be caused not only by the variation of the orientational variable $\theta$ at the considered point, but also the variations at other spatial points of the design domain. Strictly speaking, the equation $\partial u / \partial \theta=0$ corresponding to (29) cannot be solved only at a separated special point of the design domain, if the problem is to obtain the extreme strain energy corresponding to the whole design domain (not only the extreme strain energy density at a special point). Especially when one uses a discretization technique, e.g. the finite element method, for solving the problem, the coupling will exist among the discrete orientational variables that correspond to the various finite elements. Futhermore, due to the aforementioned limitation, Pedersen's method also needs to be extended to deal with more general optimization problems in which the objective functions are not as simple as the strain energy density.

\subsection{Principal stress direction method}

Suzuki and Kikuchi (1991) considered the use of the principal stress direction as the optimal orientation for the OMD problem. This consideration has a straightforward physical meaning and it is relatively simple in calculation. It is easy to understand that if the stress field is constant with respect to the orientational variable, the principal material axis should be oriented to be colinear with the principal stress direction for obtaining the stiffest structure. This method is highly efficient for the problem since the principal stresses can be easily determined. However, the method cannot be extended to solve a multicriteria optimization problem, such as the multi-loading problem discussed by Díaz and Bendsøe (1992) and also lacks a mathematical basis.

\subsection{Stress based method for the multi-loading case}

Diaz and Bendsøe (1992) extended the principal stress direction method for determining the optimal orientation in a static stiffness optimization problem corresponding to multiple loads. In this approach, first the principal stress directions are determined with respect to all loading cases, then a combined equation using these principal stress directions is solved to determine the optimal orientation. The basic equation obtained by Díaz and Bendsøe (1992) for the optimal orientation is

$\sum_{i=1}^{p} w_{i}\left[A_{e}^{i} \sin 2\left(\theta_{e}-\alpha_{e}^{i}\right)+B_{e}^{i} \sin 4\left(\theta_{e}-\alpha_{e}^{i}\right)\right]=0$,

where $\theta_{e}$ stands for the discretized orientational variable with respect to the element $e, \alpha_{e}^{i}$ the angle between the principal stress direction and the fixed reference frame for the $i$-th load case with respect to the element $e, w_{i}$ the given weighting function, $A_{e}^{i}$ and $B_{e}^{i}$ the coefficients calculated by the material constants and known stresses, and $p$ the total number of loading cases. Apparently, this approach works well for the problem discussed by Diaz and Bendsøe (1992). However, it has not been explained why it is necessary to use a stress based approach for the problem, or how this approach can be extended to general optimization problems, such as the multi-eigenvalue optimization problems discussed by $\mathrm{Ma}$ et al. (1992). In this paper, we shall propose a more general approach, in which a general stress tensor is used instead of the principal stress and it will be shown how this approach can be applied to solve the optimization problem corresponding to a dynamic system. 
5 A general approach for determining the optimal orientation

\subsection{Approach for the static problem}

First, we consider the static problem discussed previously. Using the matrix expression, the objective function meancompliance defined in (16) can be rewritten as

$\Pi=\int_{\Omega} \varepsilon^{T} \mathbf{D}^{H} \varepsilon \mathrm{d} \Omega$,

where $\varepsilon$ stands for the strain vector, i.e.

$\varepsilon=\left\{\varepsilon_{11}, \varepsilon_{22}, \varepsilon_{12}\right\}^{T}$ and $\varepsilon_{i j}=\frac{1}{2}\left(\frac{\partial u_{i}^{0}}{\partial x_{j}}+\frac{\partial u_{j}^{0}}{\partial x_{i}}\right)$.

Substituting (13) into (32) gives

$\Pi=\int_{\Omega}\left(a_{0}+a_{1} \sin 2 \theta+a_{2} \cos 2 \theta+a_{3} \sin 2 \theta \cos 2 \theta-\right.$

$\left.a_{4} \sin ^{2} 2 \theta\right) \mathrm{d} \Omega$,

where,

$a_{0}=\varepsilon^{T} \mathbf{D}^{0} \varepsilon=\frac{1}{2}\left(E_{1111}^{h}+E_{2222}^{h}\right)\left(\varepsilon_{11}^{2}+\varepsilon_{22}^{2}\right)+$

$2 E_{1122}^{h} \varepsilon_{11} \varepsilon_{22}+E_{1212}^{h} \varepsilon_{12}^{2}$,

$a_{1}=\varepsilon^{T} \mathbf{D}^{1} \varepsilon=2 d_{1}\left(\varepsilon_{11}+\varepsilon_{22}\right) \varepsilon_{12}$,

$a_{2}=\varepsilon^{T} \mathbf{D}^{2} \varepsilon=2 d_{1}\left(\varepsilon_{11}^{2}-\varepsilon_{22}^{2}\right)$,

$a_{3}=\varepsilon^{T} \mathbf{D}^{3} \varepsilon=2 d_{2}\left(\varepsilon_{11}-\varepsilon_{22}\right) \varepsilon_{12}$,

$a_{4}=\varepsilon^{T} \mathrm{D}^{4} \varepsilon=d_{2}\left[\left(\varepsilon_{11}-\varepsilon_{22}\right)^{2}-\varepsilon_{12}^{2}\right]$.

It should be noted that the coefficients $a_{i}(i=0,1, \ldots, 4)$ $a_{i}(i=0,1, \ldots, 4)$ are functions of the strain field which, in general, is a function of the orientational variable.

Using (20), the sensitivity of the mean-compliance can be rewritten as

$\frac{\partial \Pi}{\partial \theta}=-\int_{\Omega} \varepsilon \frac{\partial \mathbf{D}^{H}}{\partial \theta} \varepsilon \mathrm{d} \Omega$.

From (13), we have

$\frac{\partial \mathbf{D}^{H}}{\partial \theta}=2\left(\mathbf{D}^{1} \cos 2 \theta-\mathbf{D}^{2} \sin 2 \theta+\mathbf{D}^{3} \cos 4 \theta-\mathbf{D}^{4} \sin 4 \theta\right)$

Substituting (37) into (36) gives

$\frac{\partial \Pi}{\partial \theta}=$

$-2 \int_{\Omega}\left(a_{1} \cos 2 \theta-a_{2} \sin 2 \theta+a_{3} \cos 4 \theta-a_{4} \sin 4 \theta\right) \mathrm{d} \Omega$.

If the principal strain is considered for the problem, then replacing the strain field by $\varepsilon_{11}=\varepsilon_{I}, \varepsilon_{22}=\varepsilon_{I I}$ and $\varepsilon_{12}=0$, where $\varepsilon_{I}$ and $\varepsilon_{I I}\left(\varepsilon_{I} \geq \varepsilon_{I I}\right)$ are the principal strain, (38) is simplified to

$\frac{\partial \Pi}{\partial \theta}=-\int_{\Omega}\left[4 d_{1}\left(\varepsilon_{I}^{2}-\varepsilon_{I I}^{2}\right) \sin 2 \theta+2 d_{2}\left(\varepsilon_{I}-\varepsilon_{I I}\right)^{2} \sin 4 \theta\right] \mathrm{d} \Omega=$ $-4 \int_{\Omega}\left(\varepsilon_{I}-\varepsilon_{I I}\right) \sin 2 \theta\left[d_{1}\left(\varepsilon_{I}+\varepsilon_{I I}\right)+\right.$
$\left.d_{2}\left(\varepsilon_{I}-\varepsilon_{I I}\right) \cos 2 \theta\right] \mathrm{d} \Omega$.

This is the same result as that obtained by Pedersen (1988), i.e. (29), when the strain energy density is considered in the problem where $I I=2 \int_{\Omega} u \mathrm{~d} \Omega$.

In order to solve (28) corresponding to (38) or (39), the finite element method can be employed to discretize the problem. Defining $\theta_{e}$ as a discretized orientational design variable corresponding to the finite element $\Omega_{e}$, the optimality criteria for $\theta_{e}$ can be obtained as

$a_{1}^{e} \cos 2 \theta_{e}-a_{2}^{e} \sin 2 \theta_{e}+a_{3}^{e} \cos 4 \theta_{e}-a_{4}^{e} \sin 4 \theta_{e}=0$

$\left(e=1,2, \ldots, n_{e \ell}\right)$,

where, $n_{c \ell}$ is the total number of the finite elements in the structure,

$a_{1}^{e}=2 d_{1} \int_{\Omega_{e}}\left(\varepsilon_{11}+\varepsilon_{22}\right) \varepsilon_{12} \mathrm{~d} \Omega$,

$a_{2}^{e}=2 d_{1} \int_{\Omega_{e}}\left(\varepsilon_{11}^{2}-\varepsilon_{22}^{2}\right) \mathrm{d} \Omega$

$a_{3}^{e}=2 d_{2} \int_{\Omega_{e}}\left(\varepsilon_{11}-\varepsilon_{22}\right) \varepsilon_{12} \mathrm{~d} \Omega$,

$a_{4}^{e}=d_{2} \int_{\Omega_{e}}\left[\left(\varepsilon_{11}-\varepsilon_{22}\right)^{2}-\varepsilon_{12}^{2}\right] \mathrm{d} \Omega$.

It should be noted that (40) must be solved for all elements $\Omega_{e}\left(e=1,2, \ldots, n_{e \ell}\right)$. Basically the coupling effects may exist among these equations because coefficients $a_{i}^{e}\left(i=1,2,3,4 ; e=1,2, \ldots, n_{e \ell}\right)$ in (41), in general, are implicit functions of the discrete orientational variables $\theta_{e}(e=$ $\left.1,2, \ldots, n_{e \ell}\right)$. Therefore, a solver for these coupled nonlinear equations may be needed for the problem.

\subsection{Extension for the eigenfrequency optimization problem}

The above approach can be extended to deal with more general optimization problems. Here we consider the multieigenvalue optimization problem discussed by $\mathrm{Ma}$ et al. (1992a) as a practice of the new approach. Obviously, this approach can be applied to the other problems, e.g. the frequency response optimization problems discussed by Ma et al. (1992b).

The objective function proposed by Ma et al. (1992a) for the multi-eigenvalue optimization problem is

$\lambda^{*}=\lambda_{0}+\left[\sum_{i=1}^{m} w_{i}\left(\lambda_{n_{i}}-\lambda_{0_{i}}\right)^{n} / \sum_{i=1}^{m} w_{i}\right]^{\frac{1}{n}} \quad(n \neq 0)$,

where $\lambda^{*}$ is called the mean-eigenvalue, and $\lambda_{n_{i}}(i=1,2, \ldots$, $m)$ stand for the chosen eigenvalues that satisfy the eigenvalue problem (22).

The sensitivity of the mean-eigenvalue with respect to the orientational variable $\theta$ is obtained as

$\frac{\partial \lambda^{*}}{\partial \theta}=\frac{\left(\lambda^{*}-\lambda_{0}\right)^{1-n}}{\sum_{i=1}^{m} w_{i}} \sum_{i=1}^{m} \frac{w_{i}}{\left(\lambda_{n_{i}}-\lambda_{0_{i}}\right)^{1-n}} \frac{\partial \lambda_{n_{i}}}{\partial \theta} \quad(n \neq 0)$.

Using (25), the strain field and the finite element discretization, (43) can be discretized as 
$\frac{\partial \lambda^{*}}{\partial \theta_{e}}=$

$\frac{\left(\lambda^{*}-\lambda_{0}\right)^{1-n}}{\sum_{i=1}^{m} w_{i}} \sum_{i=1}^{m} \frac{w_{i}}{\left(\lambda_{n_{i}}-\lambda_{0_{i}}\right)^{1-n}} \int_{\Omega_{e}} \varepsilon^{n_{i}^{T}} \frac{\partial \mathrm{D}^{H}}{\partial \theta_{e}} \varepsilon^{n_{i}} \mathrm{~d} \Omega$,

where $\varepsilon^{n_{i}}=\left\{\varepsilon_{11}^{n_{i}}, \varepsilon_{22}^{n_{i}}, \varepsilon_{12}^{n_{i}}\right\}^{T}$ is the equivalent strain vector corresponding to the $n_{i}$-th eigenvector, i.e.

$\varepsilon_{r s}^{n_{i}}=\frac{1}{2}\left(\frac{\partial \phi_{r}^{n_{i}}}{\partial x_{s}}+\frac{\partial \phi_{s}^{n_{i}}}{\partial x_{r}}\right)$.

Substituting (37) into (44) obtains

$\frac{\partial \lambda^{*}}{\partial \theta_{e}}=2\left(a_{e}^{1} \cos 2 \theta_{e}-a_{e}^{2} \sin 2 \theta_{e}+a_{e}^{3} \cos 4 \theta_{e}-a_{e}^{4} \sin 4 \theta_{e}\right),($

where

$a_{e}^{k}=\frac{\left(\lambda^{*}-\lambda_{0}\right)^{1-n}}{\sum_{i=1}^{m} w_{i}} \sum_{i=1}^{m} \frac{w_{i}}{\left(\lambda_{n_{i}}-\lambda_{0_{i}}\right)^{1-n}} \int_{\Omega_{e}} \varepsilon^{n_{i}^{T}} \mathbf{D}^{k} \varepsilon^{n_{i}} \mathrm{~d} \Omega$

$(k=1,2,3,4)$.

Then the optimality criteria with respect to a discretized orientation design variable $\theta_{e}$ become,

$a_{e}^{1} \cos 2 \theta_{e}-a_{e}^{2} \sin 2 \theta_{e}+a_{e}^{3} \cos 4 \theta_{e}-a_{e}^{4} \sin 4 \theta_{e}=0$,

where $-\pi / 2 \leq \theta_{\epsilon} \leq \pi / 2$.

\subsection{Using the stress field for the approach}

It will be discussed in the examples that the stress based formulation provides better solutions than the strain based approach for the optimal orientation problem if (46) is solved separately with respect to each individual orientational variable. Hence, instead of the equivalent strain, the equivalent stress will be considered for the problem. Using the constitutive law

$\varepsilon=\mathbf{C}^{H} \sigma$,

where $\sigma$ stands for the equivalent stress vector and $\mathbf{C}^{H}=$ $\left(\mathbf{D}^{H}\right)^{-1},(44)$ can be rewritten using the equivalent stress as $\frac{\partial \lambda^{*}}{\partial \theta_{e}}=$

$-\frac{\left(\lambda^{*}-\lambda_{0}\right)^{1-n}}{\sum_{i=1}^{m} w_{i}} \sum_{i=1}^{m} \frac{w_{i}}{\left(\lambda_{n_{i}}-\lambda_{0_{i}}\right)^{1-n}} \int_{\Omega_{e}} \sigma^{n_{i}^{T}} \frac{\partial \mathbf{C}^{H}}{\partial \theta_{e}} \sigma^{n_{i}} \mathrm{~d} \Omega$.

The rotated homogenized compliance matrix $\mathbf{C}^{H}$ can be written as

$\mathbf{C}^{H}=\mathbf{T}\left(-\theta_{e}\right) \mathbf{C}^{h} \mathbf{T}^{T}\left(-\theta_{e}\right)$,

where $\mathbf{T}$ stands for the rotation matrix:

$T=\left[\begin{array}{ccc}\cos ^{2} \theta_{e} & \sin ^{2} \theta_{e} & \cos \theta_{e} \sin \theta_{e} \\ \sin ^{2} \theta_{e} & \cos ^{2} \theta_{e} & -\cos \theta_{e} \sin \theta_{e} \\ -2 \cos \theta_{e} \sin \theta_{e} & 2 \cos \theta_{e} \sin \theta_{e} & \cos ^{2} \theta_{e}-\sin ^{2} \theta_{e}\end{array}\right]$,

and

$\mathbf{C}^{h}=\left[\begin{array}{ccc}C_{1111}^{h} & C_{1122}^{h} & 0 \\ C_{1122}^{h} & C_{2222}^{h} & 0 \\ 0 & 0 & C_{1212}^{h}\end{array}\right]=\left(\mathbf{D}^{h}\right)^{-1}$.
Substituting (52) and (53) into (51), yields

$\mathbf{C}^{H}=\mathbf{C}^{0}+\mathbf{C}^{\mathbf{1}} \sin 2 \theta_{e}+\mathbf{C}^{2} \cos 2 \theta_{e}+$

$\mathrm{C}^{3} \sin 2 \theta_{e} \cos 2 \theta_{e}-\mathrm{C}^{4} \sin ^{2} 2 \theta_{e}$,

where $\mathbf{C}^{k}(k=0,1, \ldots, 4)$ are independent of the orientational variable $\theta_{e}$,

$\mathbf{C}^{0}=$

$\left[\begin{array}{ccc}\left(C_{1111}^{h}+C_{2222}^{h}\right) / 2 & C_{1122}^{h} & 0 \\ C_{1122}^{h} & \left(C_{1111}^{h}+C_{2222}^{h}\right) / 2 & 0 \\ 0 & 0 & C_{1212}^{h}\end{array}\right]$,

$\mathbf{C}^{1}=c_{1}\left[\begin{array}{lll}0 & 0 & 1 \\ 0 & 0 & 1 \\ 1 & 1 & 0\end{array}\right]$,

$\mathbf{C}^{2}=c_{1}\left[\begin{array}{ccc}1 & 0 & 0 \\ 0 & -1 & 0 \\ 0 & 0 & 0\end{array}\right]$,

$\mathbf{C}^{3}=2 c_{2}\left[\begin{array}{ccc}0 & 0 & 1 \\ 0 & 0 & -1 \\ 1 & -1 & 0\end{array}\right]$,

$\mathbf{C}^{4}=c_{2}\left[\begin{array}{ccc}1 & -1 & 0 \\ -1 & 1 & 0 \\ 0 & 0 & -4\end{array}\right]$

and $c_{1}$ and $c_{2}$ are the invariant material parameters

$c_{1}=\frac{1}{2}\left(C_{1111}^{h}-C_{2222}^{h}\right)$,

$c_{2}=\frac{1}{4}\left(C_{1111}^{h}+C_{2222}^{h}-2 C_{1122}^{h}-C_{1212}^{h}\right)$.

Thus, the sensitivity of the compliance matrix $\mathbf{C}^{H}$ with respect to $\theta_{e}$ is

$\frac{\partial \mathbf{C}^{H}}{\partial \theta_{e}}=$

$2\left(\mathbf{C}^{1} \cos 2 \theta_{e}-\mathbf{C}^{2} \sin 2 \theta_{e}+\mathbf{C}^{3} \cos 4 \theta_{e}-\mathbf{C}^{4} \sin 4 \theta_{e}\right)$.

Then the sensitivity of the objective function for the eigenvalue optimization problem can be obtained as

$\frac{\partial \lambda^{*}}{\partial \theta_{e}}=2\left(g_{e}^{1} \cos 2 \theta_{e}-g_{e}^{2} \sin 2 \theta_{e}+g_{e}^{3} \cos 4 \theta_{e}-g_{e}^{4} \sin 4 \theta_{e}\right)$,

where

$g_{e}^{k}=-\frac{\left(\lambda^{*}-\lambda_{0}\right)^{1-n}}{\sum_{i=1}^{m} w_{i}} \sum_{i=1}^{m} \frac{w_{i}}{\left(\lambda_{n_{i}}-\lambda_{0_{i}}\right)^{1-n}} \int_{\Omega_{e}} \sigma^{n_{i}^{T}} \mathbf{C}^{k} \sigma^{n_{i}} \mathrm{~d} \Omega$

(for $k=1,2,3,4$ ),

Hence, the optimality criteria for the optimal orientations $\theta_{e}$ can be obtained as

$g_{e}^{1} \cos 2 \theta_{e}-g_{e}^{2} \sin 2 \theta_{e}+g_{e}^{3} \cos 4 \theta_{e}-g_{e}^{4} \sin 4 \theta_{e}=0$,

where $-\pi / 2 \leq \theta_{e} \leq \pi / 2$ and $e=1,2, \ldots, n_{e \ell}$.

If the coefficients $g_{e}^{k}(k=1,2, \ldots, 4)$ are assumed to be constant, or they are not sensitive to the change of the discretized orientational variable, then (60) can be approximately solved separately for each individual orientational variable. Assuming $z=\tan \theta_{e},(60)$ can be transformed into the 4th-order polynomial equation as follows: 
$f_{4} z^{4}+f_{3} z^{3}+f_{2} z^{2}+f_{1} z+f_{0}=0$

where

$f_{4}=-g_{e}^{1}+g_{e}^{3}$,

$f_{3}=-2 g_{e}^{2}+4 g_{e}^{4}$,

$f_{2}=-6 g_{e}^{3}$,

$f_{1}=-2 g_{e}^{2}-4 g_{e}^{4}$,

$f_{0}=g_{e}^{1}+g_{e}^{3}$,

Solving (61), we may obtain several solutions which are real numbers. In order to determine a unique solution which is optimum for the problem, we can define an evaluation function

$\psi_{e}\left(\theta_{e}\right)=g_{e}^{1} \sin 2 \theta_{e}+g_{e}^{2} \cos 2 \theta_{e}+$

$g_{e}^{3} \sin 2 \theta_{e} \cos 2 \theta_{e}-g_{e}^{4} \sin ^{2} 2 \theta_{e}$.

By comparing the values of the evaluation function with respect to all solutions of $(60)$ and $\theta_{e}= \pm \pi / 2$, respectively, we can determine a unique solution which gives the largest value (for the maximization problem, otherwise the smallest value for the minimization problem) of the evaluation function $\psi_{e}\left(\theta_{e}\right)$ for the problem. If there is no real solution for (61), then one just needs to compare the values of the evaluation function at $\theta_{e}=-\pi / 2$ and $\theta_{e}=\pi / 2$ and choose longer one as the optimal solution.

\section{Numerical examples}

Several examples are presented in this section to verify the previous discussion and substantiate the new approach presented in this paper. First, in order to compare the results obtained by the different methods, two eigenvalue optimization problems, a single eigenvalue optimization problem and a multi-eigenvalue optimization problem, are calculated for a beam-like structure. Second, the coupling effects between the discrete orientational variables are investigated using the "Von Mises strain" and Von Mises stress. Finally, two more complicated plane stress problems are calculated using various methods, i.e. the strain based method, the principal stress direction method, the stress based method suggested in this paper, a mathematical programming method (the conjugate gradient method with a line search technique), as well as the fixed orientation method, in order to demonstrate the advantages of the method suggested. Note that in the strain based method and the stress based method, (48) and (60) are asssumed to be solved separately for an individual discretized orientational variable.

\subsection{Example 1. Optimal orientation}

This example is to show the results obtained by different methods, i.e. the principal strain direction method, the general strain based method, the principal stress direction method and the general stress based method suggested in this paper. The strain based method is identified as Pedersen's method in case 1 of this example. As shown in Fig. 3, a beam-like design domain is clamped at two ends with a concentrated mass (5.0E-06) acting at the centre of the design domain. The design domain is discretized into $2 \times 10$ finite elements. Twenty discrete orientational variables $\theta_{e}(e=1,2, \ldots, 20)$ are then defined for the 20 elements respectively, while the initial values of these orientational variables are assumed to be zero. The total volume constraint of the material is given as 3.0. Two eigenfrequency optimization problems, a single eigenvalue optimization problem (SEO) and a multi-eigenvalue optimization problem (MEO), are considered.

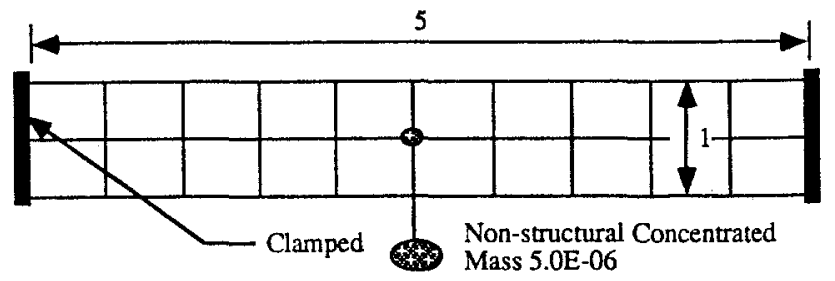

Fig. 3. Design domain for a beam-like structure

The sizing variables $a$ and $b$, which stand for the dimensions of the inside cavity of the microstructure, are assumed to be constant, 0.13 and 0.33 respectively. Then the orientational variables $\theta_{e}(e=1,2, \ldots, 20)$ are updated using the methods discussed in this paper. Note that just one step of the update is considered here.

\subsubsection{Results in single eigenvalue optimization (SEO)}

This SEO problem is to maximize the first eigenvalue of the structure. Figure 4 shows the one-step updated orientations obtained by using the following different methods, i.e. the principal strain direction method, the strain based method, the principal stress direction method, and the stress based method suggested in this paper. It is shown that, for the single eigenvalue optimization case, the result obtained by the strain based method is identical to that of the principal strain direction method, while the result obtained by the stress based method is identical to that of the principal stress direction method. However, the result obtained by the strain based method (including the principal strain direction method) is very different from that of the stress based method (including the principal stress direction method). The objective functions obtained by the aforementioned methods are $\lambda^{*}=77.2,77.2,101.8$, and 101.8 respectively, while the initial value of the objective function is 39.5. It is seen that the stress based approach can provide much better results for the problem in comparison with the use of the strain based approach.

Figure 5 shows the convergence histories of the objective functions using the different methods as the optimization process is kept going until the 31st step. It is also shown that the result obtained by the strain based method is identical to that of the principal strain direction method, and the result obtained by the stress based method is identical to that of the principal stress direction method. Furthermore, the stress based approach can provide much better results in comparison with the strain based approach.

\subsubsection{Results in multi-eigenvalue optimization (MEO)}

Three eigenvalues, the second, third and fourth eigenvalues, are considered for the objective function defined in (42), where the weighting functions corresponding to these eigenvalues are assumed to be the same, i.e. 1.0 and power $n$ is assumed to be -1 . The initial values of the sizing variables $a$ and $b$ are assumed to be 0.33 while the initial values of 


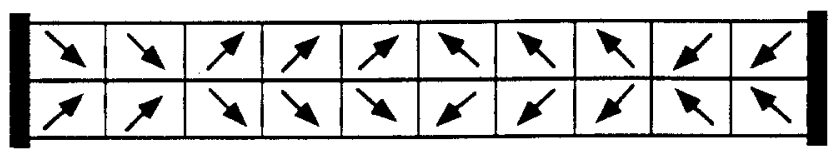

(a) Using the principal strain direction method.

$\left(\lambda^{*}=77.2\right)$

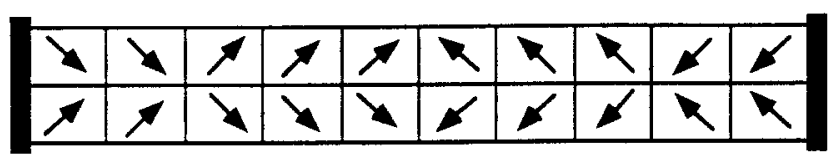

(b) Using the strain based method.

$\left(\lambda^{*}=77.2\right)$

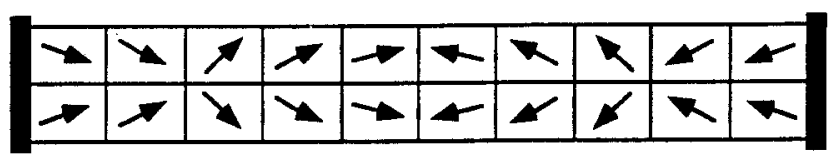

(c) Using the principal stress direction method.

$\left(\lambda^{*}=101.8\right)$

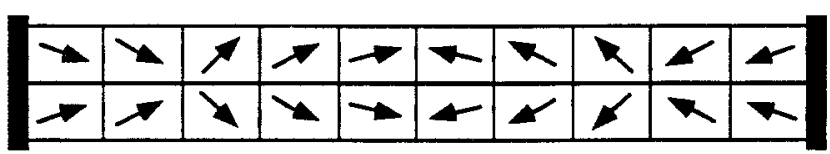

(d) Using the stress based method.

$$
\left(\lambda^{*}=101.8\right)
$$

Fig. 4. The optimal orientations by using the different methods for the SEO problem: (a) using the principal strain direction method $\left(\lambda^{*}=77.2\right)$, (b) using the strain based method $\left(\lambda^{*}=\right.$ $77.2)$, (c) using the principal stress direction method $\left(\lambda^{*}=101.8\right)$, (d) using the stress based method $\left(\lambda^{*}=101.8\right)$

the orientational variables are assumed to be zero. Figure 6 shows the final results (after 30 iterations) of the optimal orientations obtained by using different methods, where for the principal stress direction method, the principal direction is approximately determined by the second eigenmode. Since the second eigenfrequency dominates the optimization process. The objective functions obtained by the aforementioned methods, i.e. the strain based method, the principal stress direction method and the stress based method, are $323.0,358.6$ and 387.7 in comparison with the initial value of 271.3. It is shown that the use of the stress based method, which is suggested in this paper, can give much better results than the other methods. As shown in Table 1, the second eigenfrequency obtained by using the principal stress direction method has the highest value in comparison with that of using the other methods. The reason is that the principal stress directions are determined by the second eigenmode. Also, it is seen that using an approximated principal stress direction method can result in quite good objective functions

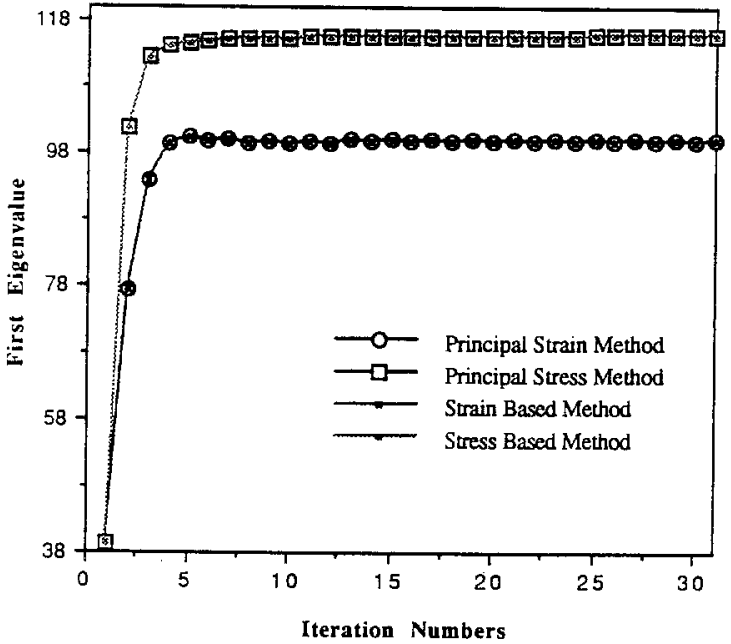

Fig. 5. The convergence history of the 1st eigenfrequency

in this problem because the second eigenmode dominates the whole process in this optimization. However, in a general multi-eigenvalue optimization problem, it is possible that no eigenmode can dominate the optimization process. In that case, the use of the principal stress direction method may result in a bad solution.

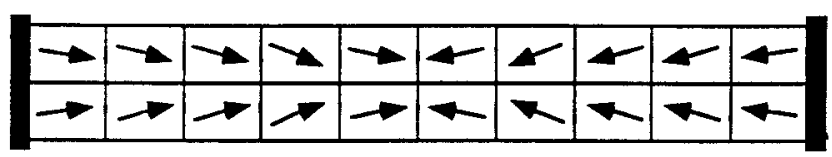

(a) Using the strain based method.

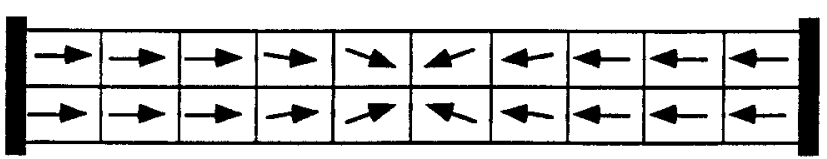

(b) Using the principal stress direction method.

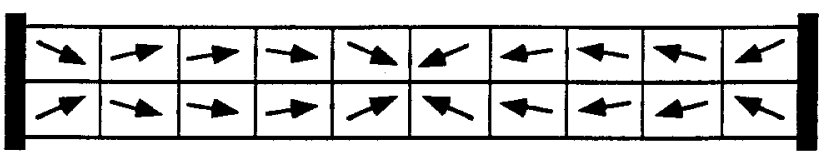

(c) Using the stress based method.

Fig. 6. Optimal orientations by using different methods for the MEO problem: (a) the strain based method, (b) the principal stress direction method, (c) the stress based method

Figure 7 shows the convergence histories obtained by the aforementioned methods. It is shown that large oscillations occur in the optimization process when the principal stress direction method is used and a relatively serious oscillation occurs when the strain based method is used. However, the use of the stress based method presented in this paper makes it possible to obtain much better results in addition with very smooth convergence history. 
Table 1. The final eigenfrequencies and objectives for the MEO problem

\begin{tabular}{|c|c|c|c|c|}
\hline & $\begin{array}{c}\text { 2nd } \\
\text { frequency }\end{array}$ & $\begin{array}{c}\text { 3rd } \\
\text { frequency }\end{array}$ & $\begin{array}{c}\text { 4th } \\
\text { frequency }\end{array}$ & Objective \\
\hline $\begin{array}{c}\text { Strain based } \\
\text { method }\end{array}$ & 240.2 & 375.1 & 481.6 & 323.0 \\
$(\mathrm{~Hz})$ & $(\mathrm{Hz})$ & $(\mathrm{Hz})$ & $(\mathrm{Hz})$ \\
\hline Principal stress & 295.2 & 391.7 & 432.9 & 358.6 \\
direction method & $(\mathrm{Hz})$ & $(\mathrm{Hz})$ & $(\mathrm{Hz})$ & $(\mathrm{Hz})$ \\
\hline New method & 278.1 & 521.7 & 545.6 & 387.7 \\
& $(\mathrm{~Hz})$ & $(\mathrm{Hz})$ & $(\mathrm{Hz})$ & $(\mathrm{Hz})$ \\
\hline
\end{tabular}

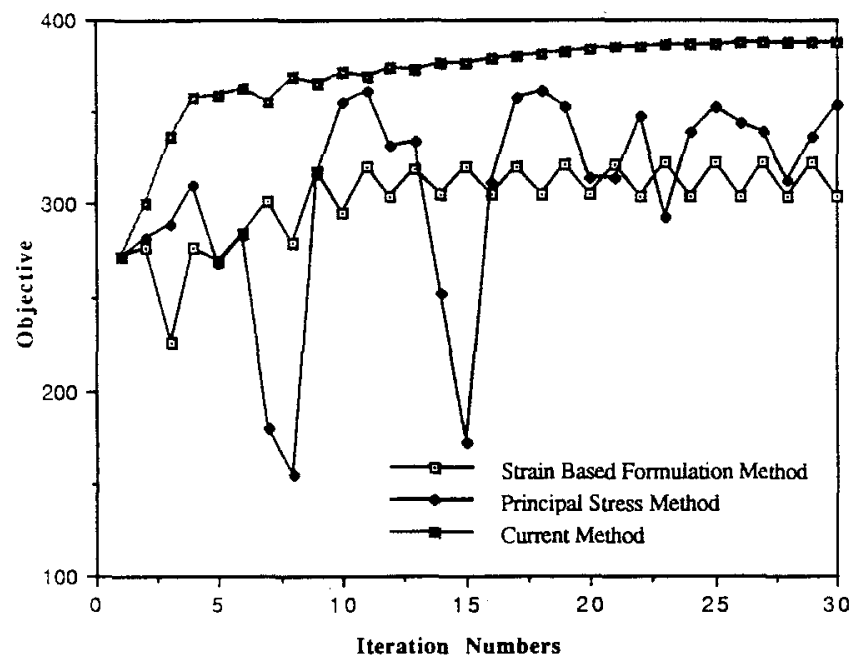

Fig. 7. Convergence history of the objective function

\subsection{Example 2. Coupling effects in discrete orientational variables}

This example is to show why the stress based method can give better results than the strain based method. For this purpose, the coupling effects among the discrete orientational variables are investigated corresponding to the strain field and the stress field. Two kinds of problem, (i) a statically determined problem and (ii) a statically undetermined problem, will be considered in this example. Figure 8 shows the design domain for the statically determined structure with simplysupported boundaries and an external force $(P=5.0)$ acting at the centre of the design domain. Figure 9 shows the design domain for the statically undetermined structure, which is almost the same as in Fig. 8, except that it is clamped at one side and roller-supported at the other side.

\begin{tabular}{|c|c|c|c|c|c|c|c|c|c|}
\hline 2 & 4 & 6 & 8 & 10 & 12 & 14 & 16 & 18 & 20 \\
\hline 1 & 3 & 5 & 7 & 9 & 11 & 13 & 15 & 17 & 19 \\
\hline
\end{tabular}

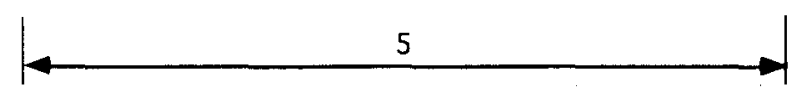

Fig. 8. Design domain of the statically determined structure

The Von Mises stress and the "Von Mises strain" are considered for the stress and strain fields respectively, where

Von Mises stress : $\sigma^{*}=\sqrt{\sigma_{11}^{2}+\sigma_{22}^{2}-\sigma_{11} \sigma_{22}+3 \sigma_{12}^{2}}$,

Von Mises strain : $\varepsilon^{*}=\sqrt{\varepsilon_{11}^{2}+\varepsilon_{22}^{2}-\varepsilon_{11} \varepsilon_{22}+3 \varepsilon_{12}^{2}}$.

\begin{tabular}{|c|c|c|c|c|c|c|c|c|c|}
\hline 2 & 4 & 6 & 8 & 10 & 12 & 14 & 16 & 18 & 20 \\
\hline 1 & 3 & 5 & 7 & 9 & 11 & 13 & 15 & 17 & 19 \\
\hline
\end{tabular}

Fig. 9. Design domain of the statically undetermined structure

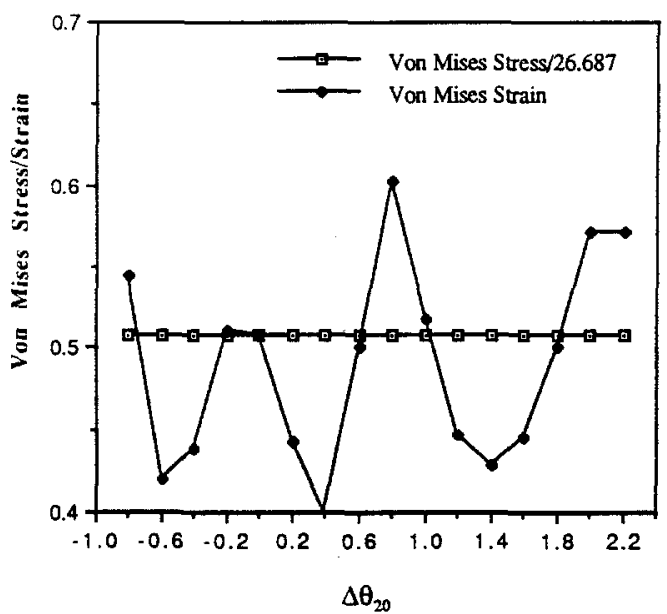

Fig. 10. Sensitivities of the von Mises stress and strain for a statically determined structure

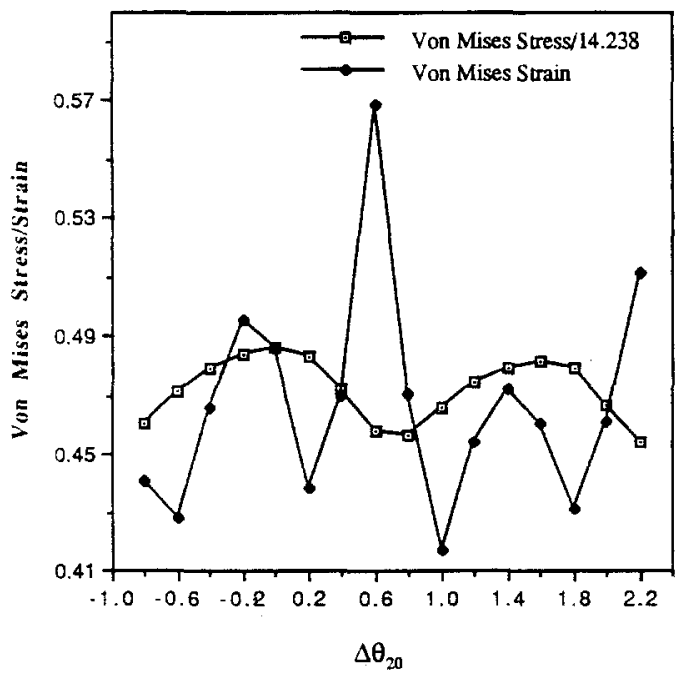

Fig. 11. Sensitivities of the von Mises stress and strain for a statically undetermined structure

In order to investigate the coupling among various elements, the orientational variable $\theta_{20}$ in the 20-th element is perturbed from -0.8 to 2.2 , while the other orientational variables are fixed. The Von Mises stress and the "von Mises strain" in the 13-th element, which are caused by the perturbation $\Delta \theta_{20}$, are typically considered. Figures 10 and 11 show the sensitivities of the Von Mises stress and the "Von Mises strain" with respect to the orientational variable. Figure 10 shows the results obtained for the statically determined structure, and Fig. 11 shows the result obtained for the statically undetermined structure, where the "Von Mises strain" is scaled to be the same dimension as the "Von Mises stress" by a constant ratio. It is shown that the strain response is much more sensitive than the stress response for 
both the statically determined and undetermined structures. It implies that much stronger coupling exists among the orientational variables when the strain field is used in comparison with the stress field. If the coupling is considerably strong, the separability of the orientational variables will no longer hold in solving the problem, i.e. (48) or (60). This explains why the stress based methods can give better results than the strain based method, which is shown in Example 1.

\subsection{Example 3. Application in topological optimization problems}

This is to show more complicated examples for the topological optimization problem. Two problems are considered here. Figure 12 shows the first problem, which is a plane structure with two clamped boundaries at the ends of the design domain and a concentrated mass at the centre of the design domain. For the second problem, as shown in Fig. 13, the design domain is clamped at the left end and simply supported at the $y$ direction of the right end, and a concentrated mass at the centre of the right end. In both problems, the finite element mesh is $20 \times 140$ with a total of 2,961 nodes. The volume constraint is given as 9.0 . Three eigenmodes, the second, third, and fourth eigenmodes, with same weights (i.e., $w_{2}=w_{3}=w_{4}=1.0$ ) are considered for the multi-eigenvalue optimization problem.

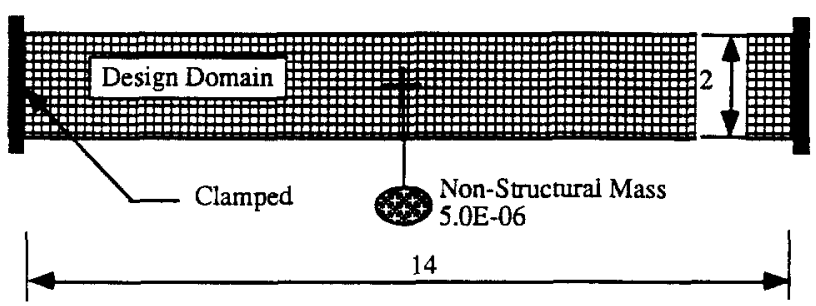

Fig. 12. Design domain for the first problem

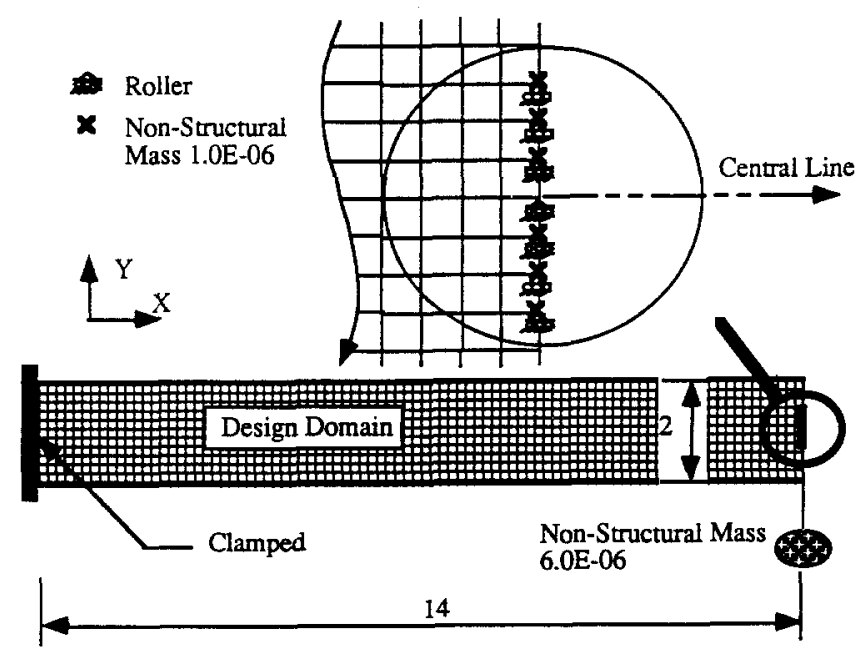

Fig. 13. Design domain for the second problem

In solving the optimal orientation problem, various methods are also employed in order to compare results. First, a special case in which the orientational variables are fixed during the optimization process is considered. Then the strain based method, the principal stress direction method and the stress based method are applied to solve the problem. Finally a mathematical programming method, the conjugate gradient method with a line search technique, is also employed for the problem. The final structures of the optimal topology designs of structures obtained by using the aforementioned methods are shown in Fig. 14 for the first problem and in Fig. 15 for the second problem. Also, the final values of both the eigenfrequencies and objective functions are listed in Table 2 for the first problem and in Table 3 for the second problem.

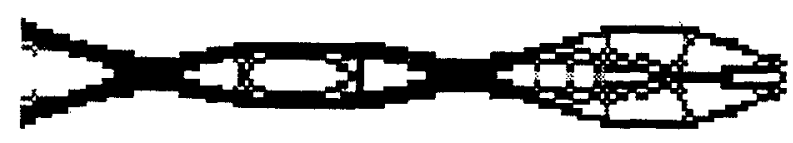

a) Fixed orientation

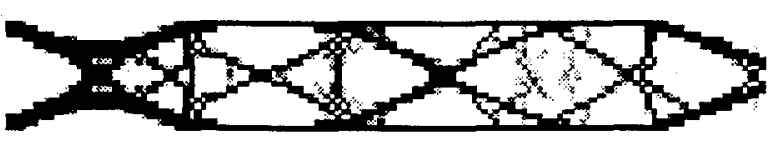

b) Using the strain based method

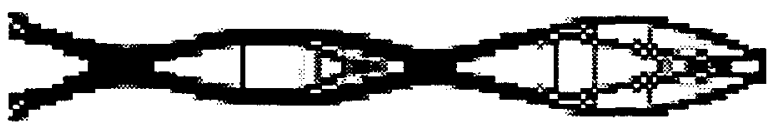

c) Using the principal stress direction method

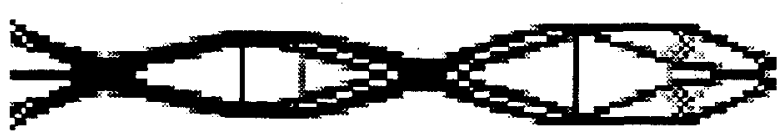

d) Using the stress based method

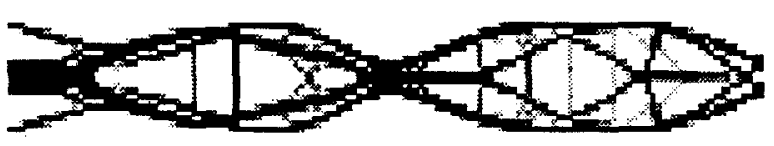

e) Using the Conjugate Gradient method

Fig. 14. The final structures for the first problem: (a) fixed orientation, (b) using the strain based method, (c) using the principal stress direction method, (d) using the stress based method, (e) using the conjugate gradient method

As shown in Fig. 14, the external shapes of the final designs internal obtained by different methods are quite similar, but the layouts are very different. The final design obtained by using the fixed orientation is not quite symmetric, and it gives a relatively lower objective function than those of the other methods except of the strain based method as shown in Table 2. The strain based method gives the worst structure and the worst objective function which even worse than that of the fixed orientation case. Although the principal stress direction method gives the maximal value of the second eigenfrequency, the suggested method provides the best objective 
function. Furthermore, the principal stress direction method may result in an unstable convergence history of the objective function as discussed in Example 1. The result obtained by the conjugate gradient method is quite similar to that of the suggested method. However, the conjugate gradient method needs a huge number of calculations to evaluate the objective function in each iteration step, which causes an unacceptable computational cost for the practical design problems. It is seen that the suggested method provides the best structure and the best objective function, and is also very efficient in calculation (similar to the principal stress direction method).

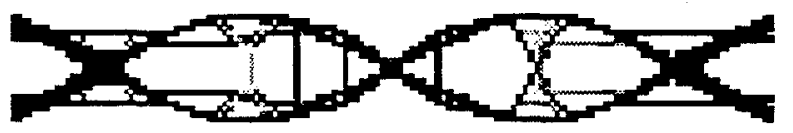

a) Fixed orientation

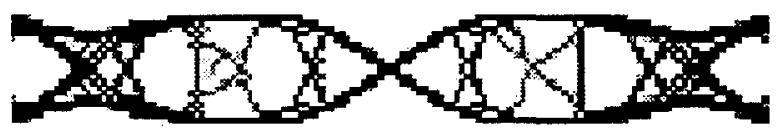

b) Using the strain based method

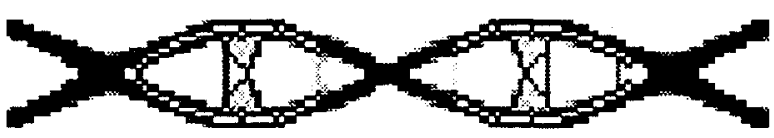

c) Using the principal stress direction method

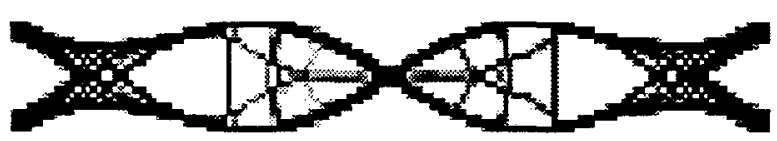

d) Using the stress based method

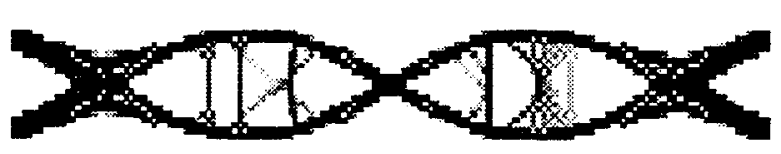

e) Using the Conjugate Gradient method

Fig. 15. The final structures for the second problem: (a) fixed orientation, (b) by the strain based method, (c) by the principal stress direction method, (d) by the stress based method, (e) by the conjugate gradient method

A similar tendency to the first problem is shown in Fig. 15 and Table 3 for the second problem. It is shown that the conjugate gradient method may give worse results than the other methods except of the strain based method. This implies that the result is dropped into lower local minima. The use of the stress based method suggested in this paper can provide the best results especially for the multimodal optimization problem.
Table 2. The final eigenfrequencies and objective tunctions for the first problem

\begin{tabular}{|c|c|c|c|c|c|}
\hline & $\begin{array}{c}\text { Fixed } \\
\text { orientation }\end{array}$ & $\begin{array}{c}\text { Strain } \\
\text { based } \\
\text { method }\end{array}$ & $\begin{array}{c}\text { Principal } \\
\text { stress } \\
\text { method }\end{array}$ & $\begin{array}{c}\text { Stress } \\
\text { based } \\
\text { method }\end{array}$ & $\begin{array}{c}\text { Conjugate } \\
\text { gradient } \\
\text { method }\end{array}$ \\
\hline $\begin{array}{c}\text { 2nd } \\
\text { eigenfrequency }\end{array}$ & $\begin{array}{c}173.3 \\
(\mathrm{~Hz})\end{array}$ & $\begin{array}{c}161.6 \\
(\mathrm{~Hz})\end{array}$ & $\begin{array}{c}180.1 \\
(\mathrm{~Hz})\end{array}$ & $\begin{array}{c}171.9 \\
(\mathrm{~Hz})\end{array}$ & $\begin{array}{c}172.5 \\
(\mathrm{~Hz})\end{array}$ \\
\hline $\begin{array}{c}\text { 3rd } \\
\text { eigenfrequency }\end{array}$ & 213.7 & 206.3 & 227.7 & 241.2 & 243.4 \\
$(\mathrm{~Hz})$ & $(\mathrm{Hz})$ & $(\mathrm{Hz})$ & $(\mathrm{Hz})$ & $(\mathrm{Hz})$ \\
\hline 4th & 229.4 & 221.0 & 235.7 & 259.0 & 246.5 \\
eigenfrequency & $(\mathrm{Hz})$ & $(\mathrm{Hz})$ & $(\mathrm{Hz})$ & $(\mathrm{Hz})$ & $(\mathrm{Hz})$ \\
\hline Objective & 201.1 & 191.0 & 209.9 & 213.3 & 211.7 \\
function & $(\mathrm{Hz})$ & $(\mathrm{Hz})$ & $(\mathrm{Hz})$ & $(\mathrm{Hz})$ & $(\mathrm{Hz})$ \\
\hline
\end{tabular}

Table 3. The final eigenfrequencies and objective functions for the second problem

\begin{tabular}{|c|c|c|c|c|c|}
\hline & \begin{tabular}{|c|} 
Fixed \\
orientation
\end{tabular} & \begin{tabular}{|c|} 
Strain \\
based \\
method
\end{tabular} & $\begin{array}{c}\text { Principal } \\
\text { stress } \\
\text { method }\end{array}$ & \begin{tabular}{|c|} 
Stress \\
based \\
method
\end{tabular} & $\begin{array}{c}\text { Conjugate } \\
\text { gradient } \\
\text { method }\end{array}$ \\
\hline $\begin{array}{c}\text { 2nd } \\
\text { eigenfrequency }\end{array}$ & $\begin{array}{l}98.04 \\
(\mathrm{~Hz})\end{array}$ & $\begin{array}{c}82.13 \\
(\mathrm{~Hz})\end{array}$ & $\begin{array}{c}97.39 \\
(\mathrm{~Hz})\end{array}$ & $\begin{array}{l}96.06 \\
(\mathrm{~Hz})\end{array}$ & $\begin{array}{l}88.08 \\
(\mathrm{~Hz})\end{array}$ \\
\hline \begin{tabular}{|c|} 
3rd \\
eigenfrequency
\end{tabular} & $\begin{array}{c}143.09 \\
(\mathrm{~Hz})\end{array}$ & $\begin{array}{c}165.96 \\
(\mathrm{~Hz})\end{array}$ & $\begin{array}{c}161.43 \\
(\mathrm{~Hz})\end{array}$ & $\begin{array}{c}169.59 \\
(\mathrm{~Hz})\end{array}$ & $\begin{array}{c}170.16 \\
(\mathrm{~Hz})\end{array}$ \\
\hline \begin{tabular}{|c|} 
4th \\
eigenfrequency \\
\end{tabular} & $\begin{array}{c}179.20 \\
(\mathrm{~Hz})\end{array}$ & $\begin{array}{c}192.11 \\
(\mathrm{~Hz})\end{array}$ & $\begin{array}{c}194.20 \\
(\mathrm{~Hz})\end{array}$ & $\begin{array}{c}199.64 \\
(\mathrm{~Hz})\end{array}$ & $\begin{array}{c}217.32 \\
(\mathrm{~Hz})\end{array}$ \\
\hline $\begin{array}{l}\text { Objective } \\
\text { function }\end{array}$ & $\begin{array}{c}127.68 \\
(\mathrm{~Hz})\end{array}$ & $\begin{array}{c}119.06 \\
(\mathrm{~Hz})\end{array}$ & $\begin{array}{c}132.72 \\
(\mathrm{~Hz})\end{array}$ & $\begin{array}{c}133.53 \\
(\mathrm{~Hz})\end{array}$ & $\begin{array}{c}127.48 \\
(\mathrm{~Hz})\end{array}$ \\
\hline
\end{tabular}

\section{Conclusion}

Several approaches for determining the optimal orientation of an orthotropic material used in the topology optimization are discussed in this paper. It is shown that the use of the strain based method may obtain even worse results than the case in which the orientations are fixed because strong coupling exists among the design variables when the strain field is used. An approximated principal stress direction method can provide quite good results for some problems, but it may cause an oscillatory convergence history of the objective function, and more importantly, it is less general. The mathematical programming method is not suggested due to its expensive calculation cost and sometime cannot give the best result for the problem. Therefore, the stress based approach is preferred for the current optimization problem. This approach not only gives the best result for the problem, but also has the generality for various optimization problems. Moreover, it is a quite efficient method. Several examples have been presented to support the issues mentioned above.

\section{References}

Bendsøe, M.P.; Kikuchi, N. 1988: Generating optimal topologies in structural design using a homogenization method. Comp. Meth. Appl. Mech. Engrg. 71, 197-24

Díaz, A.; Bendsøe, M. 1992: Shape optimization of structures for multiple loading conditions using a homogenization method. Struct. Optim. 4, 17-22

Gibiansky, L.V.; Cherkaev, A.V. 1988: Microstructures of composites of external and exact estimates of provided energy density. Technical Report, Ioffe Science and Technology Institute, Leningrad 
Guedes, J.M.; Kikuchi, N. 1990: Preprocessing and postprocessing for materials based on the homogenization method with adaptive finite element methods. Comp. Meth. Appl. Mech. Engrg. 83, 143-198

Kohn, R.V. 1990: Numerical structural optimization via a relaxed formulation. Lectures at a NATO-ASI on Free Boundary Problems and Domain Optimization

Lion, J.L. 1981: Some methods in the mathematical analysis of systems and their controls. Beijng: Science Press; New York: Gordon and Beach

Ma, Z.D.; Kikuchi, N.; Cheng, H.C.; Hagiwara, I. 1992a: Topology and shape optimization technique for structural dynamic problems. Recent Advances in Structural Problems, PVP-Vol. 248/NEVol. 10, pp. 133-143

Received Aug. 23, 1993
Ma, Z.D.; Kikuchi, N.; Hagiwara, I. 1992b: Structural topology and shape optimization for a frequency response problem. Comp. Mech. (to appear)

Olhoff, N.; Thomsen, J.; Rasmussen, J. 1992: Topology optimization of bi-material structures. Proc. IUTAM Symp. on Optimal Design with Advanced Materials

Pedersen, P. 1988: On optimal orientation of orthotropic material. Report No. 375, The Danish Center for Applied Mathematics and Mechanics, The Technical University of Denmark

Suzuki, K.; Kikuchi, N. 1991: A homogenization method for shape and topology optimization. Comp. Meth. Appl. Mech. Engrg. 93, 291-318 